\section{Changes in the prevalence of physical inactivity and sedentary behavior during COVID-19 pandemic: a survey with 39,693 Brazilian adults}

\author{
Mudanças na prevalência de inatividade física e \\ comportamento sedentário durante a pandemia \\ da COVID-19: um inquérito com 39.693 \\ adultos brasileiros
}

\section{Cambios en la prevalencia de inactividad física y comportamiento sedentario durante la pandemia de COVID-19: una encuesta con 39.693 adultos brasileños}

Danilo Rodrigues Pereira da Silva 1

André Oliveira Werneck 2

Deborah Carvalho Malta 3

Paulo Roberto Borges de Souza Júnior 4

Luiz Otávio Azevedo 4

Marilisa Berti de Azevedo Barros 5

Celia Landmann Szwarcwald 4

doi: 10.1590/0102-311X00221920

\begin{abstract}
This study analyzed changes in the prevalence of physical inactivity and sedentary behaviors according to correlates during the COVID-19 pandemic among Brazilian adults. A national retrospective online survey was conducted with 39,693 Brazilian adults. Physical activity (weekly frequency and daily duration; cut-off point of 150 minutes/week), TV-viewing time and computer/tablet use (daily duration; cut-off point of 4 hours/day) before and during the pandemic period were reported. Sex, age group, schooling level, skin color, per capita income, country region, working status during the quarantine, and adherence to the quarantine were the correlates. Descriptive statistics were used. The prevalence of physical inactivity, high TV-viewing time and computer/tablet use increased, respectively, 26\%, 266\%, and 38\% during the pandemic. While increases in physical inactivity and computer/tablet were more widespread, higher increases in the prevalence of high TV viewing tiem were observed among younger adults (660\%), with higher schooling level (437\%) and those who were at home office (331\%). The prevalence of physical inactivity and sedentary behaviors increased in all population sub-groups during the COVID-19 pandemic in Brazil.
\end{abstract}

Health Behavior; Exercise; Healthy Lifestyle; COVID-19

\author{
Correspondence \\ D. R. P. Silva \\ Departamento de Educação Física, Centro de Ciências \\ Biológicas e da Saúde, Universidade Federal de Sergipe. \\ Campus Universitário Prof. Aloísio de Campos, São Cristóvão, \\ SE 49100-000, Brasil. \\ danilorpsilva@gmail.com \\ 1 Centro de Ciências Biológicas e da Saúde, Universidade \\ Federal de Sergipe, São Cristóvão, Brasil. \\ 2 Faculdade de Saúde Pública, Universidade de São Paulo, São \\ Paulo, Brasil. \\ 3 Escola de Enfermagem, Universidade Federal de Minas Gerais, \\ Belo Horizonte, Brasil. \\ 4 Instituto de Comunicação e Informação Científica e \\ Tecnológica em Saúde, Fundação Oswaldo Cruz, Rio de Janeiro, \\ Brasil. \\ 5 Faculdade de Ciências Médicas, Universidade Estadual de \\ Campinas, Campinas, Brasil.
}




\section{Introduction}

Physical inactivity and sedentary behavior are reaching alarming levels worldwide 1 . Specifically, previous study found that approximately $55 \%$ of Brazilian adults did not attend the recommended levels of physical activity and $15 \%$ spend more than four hours per day watching TV 2 . Thus, the promotion of active lifestyles has been challenging researchers, policy makers, and international organizations 3 .

However, the new coronavirus (COVID-19) pandemic came unexpectedly and changed the human lives 4,5. Measures of quarantine to mitigate the virus spread, encouraging people to stay at their homes have been recommended as a significant measure, but it can also present indirect negative effects on healthy behaviors, reducing the opportunities to practice physical activity outside as well as increasing possibilities of sedentary activities, especially through screens (e.g., TV viewing, computer, and smartphones) 6,7 . This scenario can be a risk for cardiovascular 8 , metabolic ${ }^{9}$, and mental disorders 10 , in addition to compromise immune systems 11,12,13, directly associated to the COVID-19 progression.

In this sense, to survey the frequency and distribution of changes in movement behaviors with nationwide samples during the COVID-19 pandemic can foment and drive interventions and public policies to encourage people to stay active and with low sedentary behavior ${ }^{7}$, mitigating the harmful effects of the quarantine period. Here, we analyzed changes in the prevalence of physical inactivity and sedentary behaviors during the COVID-19 pandemic according correlates, among Brazilian adults.

\section{Methods}

\section{Design and sample}

The ConVid - Behavior Survey is a health survey using a virtual questionnaire to assess the changes that occurred in the lives of Brazilians after the arrival of the coronavirus pandemic in the country, related to social restriction initiatives for the protection of people, including quarantine. Data collection was conducted between April 24th and May 24th, 2020.

Participants were invited by a chain sampling procedure. In the first stage, the 15 members of the research team choose a total of 200 other researchers from different states in Brazil (approximately 13 invitation per member). Furthermore, members of the research team choose 20 other people from their social network, making a total of 500 people chosen $(200$ researchers +300 people of social network), which were called as influencers. Each influencer sent the survey link to at least 12 people from their social networks, obeying a stratification by sex, age range $(18-39 ; 40-59 ; 60+$ years) and education level (incomplete high school or less; complete higher education). That is, six women and six men, two in each age group, one of each education level. People invited by the influencers were encouraged to invite at least three more people of their social networks, representing the second wave of invitations). Moreover, information about the study was disseminated by press releases, social communications from participating research institutions, state health departments, and social media. The survey link was also available at the influencers' research institutions. Futher details about the study procedures are available elsewhere 14 . All procedures were approved by the Brazilian National Ethics Research Committee (CONEP) (process: 30598320.1.0000.5241). From the initial sample ( $\mathrm{n}=$ 45,160), 5,467 presented missing data in at least one variable and were excluded from the analyses. The current study excluded missing data and analyzed 39,693 participants. The sample was weighted according to characteristics from the Brazilian National Household Sample Survey (PNAD - conducted annually), considering population in each state, education, age, sex, and prevalence of chronic diseases, aiming to let the sample nationally representative.

\section{Physical activity}

For physical activity before COVID-19 pandemic, participants were asked "Before the COVID-19 pandemic, how many days a week did you practice any type of physical exercise or sport? (do not consider physical therapy)". Possible answers were: (a) less than 1; (b) 1-2; (c) 3-4; or (d) 5 or more. For those reporting physical activity practice, we also asked: "How long did this activity last?". Pos- 
sible answers were: (a) less than 30 minutes; (b) 30-45 minutes; (c) 46-60 minutes; or (d) more than 60 minutes. Furthermore, for physical activity during the COVID-19 pandemic was asked: "During the COVID-19 pandemic how many days a week did you practice any type of physical exercise or sport? (do not consider physical therapy)". Possible answers were: (a) less than 1; (b) 1-2; (c) 3-4; or (d) 5 or more. For those reporting physical activity practice, we also asked: "How long did this activity last?". Possible answers were: (a) less than 30 minutes; (b) 30-45 minutes; (c) 46-60 minutes; or (d) more than 60 minutes. Physical inactivity was classified as less than 150 minutes/week of physical activity, which was calculated using the median point of frequency and duration in each category. Physical activity practice patterns were created using four change patterns: (1) consistently active; (2) become active; (3) become low active; or (4) consistently insufficiently active.

\section{Sedentary behavior}

TV viewing and computer/tablet use were adopted as independent proxies of sedentary behavior. For TV viewing, participants were asked: "Usually, before the pandemic, how many hours a day did you used to spend watching television?" and "During the pandemic, how many hours a day have you been watching television?". For computer/tablet, participants were asked: "Usually, before the pandemic, how many hours a day did you used to spend on computer or tablet?" and "During the pandemic, how many hours a day have you been using computer or tablet?". We adopted 4 hours/day as cut-offs for high TV viewing and computer/tablet use as separately outcomes 15. TV-viewing and computer/ tablet use patterns were created using four change patterns: (1) consistently low; (2) become low; (3) become high; or (4) consistently high.

\section{Correlates}

We used gender (male/female), age group (18-29, 30-39, 40-49, 50-59, 60+ years), schooling level (none or elementary school, high school and higher education), skin color (white, black, brown, and other), per capita income ( $<1,1-2$, and $>2$ minimum wages - USD 195), and country regions (North, Northeast, Central, South, and Southeast) as sociodemographic correlates.

For correlates directly associated to the COVID-19 pandemic, we used the working status during the pandemic (normal, home office, no working for any reasons [including vacation]) and the adherence to the quarantine. This last was assessed with the question "During the Coronavirus pandemic, to what extent did you (or are you still) restricting contact with people?," with possible answers: "I did nothing, I led a normal life"; "I tried to take care, stay away from people, reduce contact a little, not visit the elderly, but I kept working and leaving”; "I stayed at home just going shopping at the supermarket and drug store" or "stayed strictly at home, leaving only for health care needs." We classified stricter adherence to the quarantine with the answers "I stayed at home just going shopping at the supermarket and drug store" or "stayed strictly at home, leaving only for health care needs". Among them, time in quarantine was also collected ( $<1$ month, 1-2 months, and $>2$ months). Those who answered "I did nothing, I led a normal life"; "I tried to take care, stay away from people, reduce contact a little, not visit the elderly, but I kept working and leaving" was classified as no adherence to the quarantine (lower impact on daily activities).

\section{Statistical procedures}

Data were analyzed using percentages and 95\% confidence intervals (95\%CI). Four mutually exclusive categories of the change pattern of physical activity, TV viewing, and computer/tablet use were separately created based on the behaviors adopted before and during the pandemic. Distribution of these behavioral categories within each population sub-group was analyzed. All statistical procedures were conducted using sampling weights (survey command) in Stata 15.1 (https://www.stata.com). 


\section{Results}

After exclusions for missing information, 39,693 participants (48\% women; 95\%CI: 46.4-49.5) composed the final sample. The proportion of participants with none or elementary school level was 9.8\% (95\%CI: 8.8-10.9) and 45.6\% (95\%CI: 44.1-47.0) were white. More than half the participants were not working during quarantine (53.4\%; $95 \%$ CI: 51.9-55.0) and $25.9 \%$ (95\%CI: $24.6-27.2)$ were at home office. Around a quarter of participants (26.2\%; 95\%CI: 24.8-27.6) reported no adherence to stricter measures of quarantine and $46.5 \%$ (95\%CI: 44.8-47.9) were between one and two months on quarantine. The prevalence of physical inactivity, $\geq 4$ hours/day of TV viewing and $\geq 4$ hours/day of computer/tablet use before and during the pandemic are presented in Figure 1.

Table 1 shows the distribution of physical activity patterns among each population sub-group. Higher proportion of "become low active" was observed among the younger age group (25\%), people with higher schooling level (28\%), and higher per capita income (28\%) compared to their counterparts. Higher proportion of "become active" was observed among people who were not working during the pandemic (4\%) and who were at home office (5\%) (compared to normal routine group), among higher per capita income group (5\%) and in the Southeast region (4\%) (compared to Central). However, regardless of changes, higher proportion of consistently insufficiently active and active was observed, respectively, among women vs. men, lower vs higher schooling level, and lower vs higher income. Furthermore, Central Region showed higher proportion of consistently insufficiently active than Northeast and Southeast regions, while the older adults group showed higher proportion of consistently active in comparison to the younger age group.

Table 2 shows the TV-viewing ( $\geq 4$ hours/day) patterns according to sub-groups. The proportion of "become high" was higher among women (30\%), 30-39 years group (31\%) (compared to $>60$ years old), white (27\%) (compared to black), among those who were not working during the pandemic (33\%) (compared to normal routine and home office groups), in Northeast (31\%) and Southeast (28\%) regions (compared to Central), among people who reported $<1$ month (29\%) and 1-2 months of quarantine (30\%) (compared to no stricter quarantine). Higher proportion of consistently high and low TV-viewing time was observed, respectively, among older vs. younger, lower vs. higher school-

Figure 1

Changes in the prevalence of movement behaviors during the COVID-19 pandemic in Brazil $(n=39,693)$.

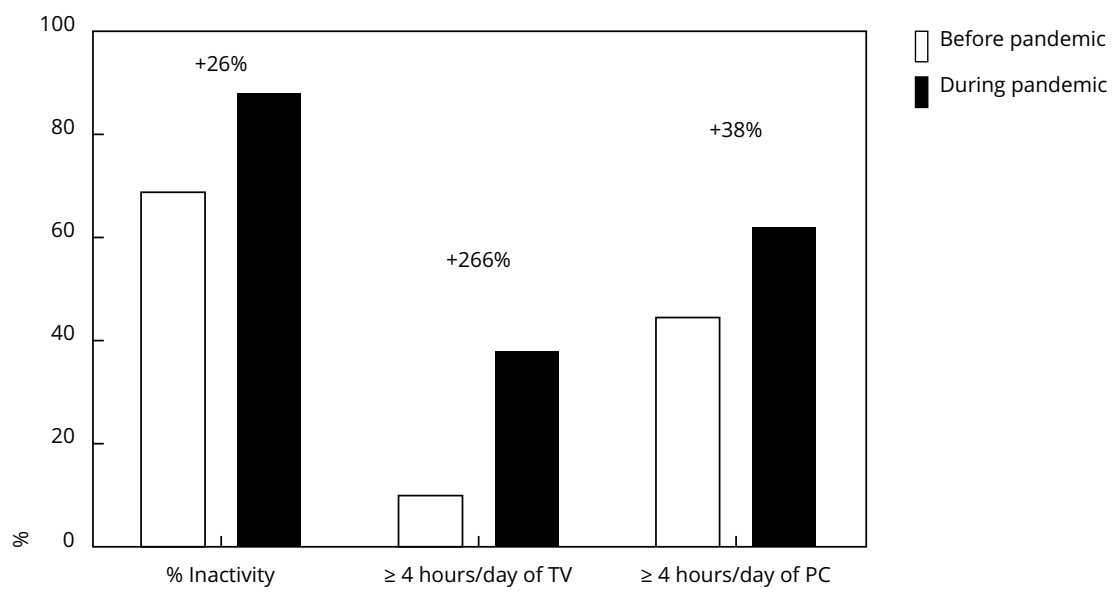

PC: personal computer or tablet.

Note: inactivity was defined as $<150$ minutes of physical activity per week. 
Table 1

Patterns of physical activity according to the correlate categories during the COVID-19 pandemic. Brazil, 2020.

\begin{tabular}{|c|c|c|c|c|}
\hline & $\begin{array}{l}\text { Consistently insufficiently active } \\
\qquad \%(95 \% \mathrm{Cl})\end{array}$ & $\begin{array}{l}\text { Become active } \\
\quad \%(95 \% \mathrm{Cl})\end{array}$ & $\begin{array}{l}\text { Become low active } \\
\quad \%(95 \% \mathrm{Cl})\end{array}$ & $\begin{array}{c}\text { Consistently active } \\
\%(95 \% \mathrm{Cl})\end{array}$ \\
\hline \multicolumn{5}{|l|}{ Gender } \\
\hline Female & $69.0(67.4-70.4)$ & $3.3(2.8-3.8)$ & $20.7(19.4-22.1)$ & $7.1(6.3-7.9)$ \\
\hline Male & $63.8(61.3-66.3)$ & $3.4(2.6-4.5)$ & $21.8(19.7-24.0)$ & $11.0(9.5-12.7)$ \\
\hline \multicolumn{5}{|l|}{ Age group (years) } \\
\hline $18-29$ & $63.7(60.9-66.3)$ & $4.3(3.5-5.3)$ & $25.1(22.7-27.6)$ & $7.0(5.8-8.4)$ \\
\hline $30-39$ & $66.3(62.9-69.5)$ & $3.0(2.1-4.1)$ & $22.6(19.9-25.7)$ & $8.0(6.3-10.2)$ \\
\hline $40-49$ & $69.8(66.4-72.9)$ & $2.6(1.5-4.3)$ & $18.3(15.9-21.1)$ & $9.3(7.4-11.1)$ \\
\hline $50-59$ & $67.0(63.9-70.0)$ & $3.3(2.3-4.7)$ & $19.0(16.7-21.6)$ & $10.7(8.9-12.3)$ \\
\hline 60 & $67.0(63.2-70.6)$ & $3.2(2.1-4.9)$ & $19.2(16.3-22.5)$ & $10.6(8.4-13.4)$ \\
\hline \multicolumn{5}{|l|}{ Schooling level } \\
\hline Less than elementary school & $76.0(70.2-80.5)$ & $2.9(1.4-5.7)$ & $13.2(9.7-17.6)$ & $8.0(5.4-11.6)$ \\
\hline High school & $67.5(65.6-69.3)$ & $3.3(2.7-4.0)$ & $20.7(19.1-22.2)$ & $8.5(7.5-9.7)$ \\
\hline Higher education & $56.9(56.2-57.6)$ & $3.9(3.6-4.1)$ & $28.2(27.5-28.8)$ & $11.1(10.6-11.5)$ \\
\hline \multicolumn{5}{|l|}{ Skin color } \\
\hline White & $65.1(63.6-66.7)$ & $3.0(2.6-3.6)$ & $22.2(21.0-23.6)$ & $9.6(8.7-10.6)$ \\
\hline Black & $65.3(60.3-70.0)$ & $4.3(2.4-7.6)$ & $20.3(16.8-24.5)$ & $10.1(7.5-13.4)$ \\
\hline Brown & $68.1(65.5-70.7)$ & $3.5(2.7-4.5)$ & $20.4(18.2-22.7)$ & $8.0(6.6-9.8)$ \\
\hline Other & $66.3(59.2-72.8)$ & $3.9(2.4-6.2)$ & $22.1(16.8-28.5)$ & $7.6(5.1-11.3)$ \\
\hline \multicolumn{5}{|l|}{ Per capita income (minimum wage) } \\
\hline$<1$ & $70.5(68.3-72.7)$ & $2.7(2.1-3.6)$ & $18.6(16.8-20.6)$ & $8.1(6.8-9.6)$ \\
\hline $1-2$ & $69.7(66.9-72.4)$ & $2.9(2.2-3.9)$ & $19.4(17.3-21.8)$ & $7.9(6.3-9.8)$ \\
\hline$>2$ & $56.3(54.0-58.7)$ & $4.8(3.8-6.0)$ & $27.5(25.5-29.7)$ & $11.3(10.1-12.7)$ \\
\hline \multicolumn{5}{|l|}{ Country region } \\
\hline North & $68.1(61.4-74.2)$ & $3.4(1.8-6.5)$ & $20.9(16.1-26.8)$ & $7.5(4.4-12.6)$ \\
\hline Northeast & $64.4(60.6-68.0)$ & $2.8(1.8-4.3)$ & $23.5(20.3-27.1)$ & $9.3(7.2-11.9)$ \\
\hline Southeast & $65.2(63.7-66.7)$ & $3.9(3.3-4.7)$ & $21.1(19.9-22.4)$ & $9.7(8.8-10.8)$ \\
\hline South & $68.7(65.2-72.1)$ & $3.1(2.0-4.9)$ & $20.4(17.7-23.5)$ & $7.7(5.9-10.0)$ \\
\hline Central & $76.2(70.1-81.4)$ & $1.6(0.9-2.8)$ & $15.7(11.7-20.8)$ & $6.4(4.1-10.0)$ \\
\hline \multicolumn{5}{|l|}{ Working during the pandemic } \\
\hline No & $66.2(64.2-68.2)$ & $3.6(3.0-4.4)$ & $21.6(19.9-23.4)$ & $8.6(7.4-9.9)$ \\
\hline Normal routine & $69.1(65.8-72.2)$ & $1.5(0.9-2.2)$ & $19.8(17.3-22.6)$ & $9.6(7.7-11.9)$ \\
\hline Home office & $65.0(62.3-67.5)$ & $4.2(3.2-5.7)$ & $21.6(19.6-23.8)$ & $9.1(7.7-10.7)$ \\
\hline \multicolumn{5}{|c|}{ Adherence to the quarantine (months) } \\
\hline No & $67.2(64.2-70.1)$ & $2.5(1.7-3.7)$ & $20.1(17.7-22.8)$ & $10.1(8.3-12.3)$ \\
\hline$<1$ & $69.7(66.1-73.1)$ & $2.7(1.7-4.1)$ & $20.6(17.6-24.0)$ & $7.0(5.5-8.8)$ \\
\hline $1-2$ & $65.0(63.1-66.8)$ & $4.1(3.3-4.9)$ & $21.9(20.4-23.4)$ & $9.1(7.9-10.4)$ \\
\hline$>2$ & $64.7(58.8-70.2)$ & $3.7(2.3-5.8)$ & $22.6(17.7-28.3)$ & $9.0(6.1-13.2)$ \\
\hline
\end{tabular}

95\%Cl: 95\% confidence interval.

ing level, no working vs. working during the pandemic, Southeast Region vs. Central. Whereas the proportion of consistently low TV-viewing time was lower among those who reported less strict adherence to quarantine measures.

Table 3 shows the patterns of computer/tablet use ( $\geq 4$ hours/day) according to population subgroups. The younger age group (27\%), people who were not working during pandemic (25\%), and those who adhered to the quarantine (23\% and $24 \%$ to 1 and $1-2$ months, respectively) presented higher proportion of "become high" computer/tablet use. Higher proportion of "become low" were 
Table 2

Patterns of TV-viewing according to the correlate categories during the COVID-19 pandemic. Brazil, 2020.

\begin{tabular}{|c|c|c|c|c|}
\hline & $\begin{array}{c}\text { Consistently high } \\
\%(95 \% \mathrm{Cl})\end{array}$ & $\begin{array}{c}\text { Become low } \\
\%(95 \% \mathrm{Cl})\end{array}$ & $\begin{array}{l}\text { Become high } \\
\%(95 \% \mathrm{Cl})\end{array}$ & $\begin{array}{c}\text { Consistently low } \\
\%(95 \% \mathrm{Cl})\end{array}$ \\
\hline \multicolumn{5}{|l|}{ Gender } \\
\hline Female & $9.2(8.1-10.4)$ & $1.0(0.7-2.4)$ & $30.2(28.7-31.8)$ & $59.6(57.9-61.3)$ \\
\hline Male & $10.0(8.4-11.6)$ & $0.5(0.2-0.9)$ & $26.0(23.8-28.3)$ & $63.7(61.1-66.2)$ \\
\hline \multicolumn{5}{|l|}{ Age group (years) } \\
\hline $18-29$ & $3.9(3.0-5.2)$ & $0.2(0.1-0.4)$ & $26.7(24.3-29.3)$ & $69.1(66.4-71.7)$ \\
\hline $30-39$ & $6.8(5.4-8.5)$ & $0.5(0.3-1.1)$ & $31.1(27.9-34.6)$ & $61.5(58.0-64.9)$ \\
\hline $40-49$ & $9.5(7.2-12.3)$ & $0.7(0.4-1.3)$ & $29.9(26.7-33.2)$ & $59.9(56.3-63.5)$ \\
\hline $50-59$ & $12.8(10.8-15.2)$ & $1.1(0.6-1.8)$ & $29.2(26.3-32.2)$ & $56.9(53.7-60.2)$ \\
\hline 60 & $17.4(14.5-20.7)$ & $1.4(0.8-2.8)$ & $24.2(21.2-27.5)$ & $57.0(53.1-60.7)$ \\
\hline \multicolumn{5}{|l|}{ Schooling level } \\
\hline Less than elementary school & $14.0(10.3-19.0)$ & $1.5(0.8-3.0)$ & $27.2(22.7-32.1)$ & $57.2(51.6-62.7)$ \\
\hline High school & $9.8(8.8-11.1)$ & $0.7(0.5-1.1)$ & $28.6(26.9-30.4)$ & $60.8(58.9-62.7)$ \\
\hline Higher education & $5.5(5.2-5.8)$ & $0.5(0.4-0.6)$ & $26.7(26.1-27.4)$ & $67.3(66.6-67.9)$ \\
\hline \multicolumn{5}{|l|}{ Skin color } \\
\hline White & $8.6(7.7-9.6)$ & $0.9(0.6-1.3)$ & $26.8(25.4-28.2)$ & $63.7(62.1-65.3)$ \\
\hline Black & $11.9(8.8-16.0)$ & $1.1(0.4-2.9)$ & $30.7(26.0-35.8)$ & $56.3(51.0-61.4)$ \\
\hline Brown & $10.0(8.4-11.9)$ & $0.6(0.3-1.0)$ & $29.1(26.7-31.6)$ & $60.3(57.5-63.0)$ \\
\hline Other & $7.6(4.0-14.2)$ & $0.3(0.1-2.0)$ & $28.8(21.6-37.3)$ & $63.2(54.7-71.0)$ \\
\hline \multicolumn{5}{|l|}{ Per capita income (minimum wage) } \\
\hline$<1$ & $9.6(8.3-11.0)$ & $0.9(0.6-1.3)$ & $28.2(26.2-30.4)$ & $61.3(59.0-63.6)$ \\
\hline $1-2$ & $10.7(8.5-13.3)$ & $0.3(0.2-0.7)$ & $28.7(26.0-31.5)$ & $60.3(57.0-63.4)$ \\
\hline$>2$ & $8.4(7.1-9.7)$ & $0.9(0.5-1.6)$ & $27.6(25.4-29.8)$ & $63.2(60.8-65.5)$ \\
\hline \multicolumn{5}{|l|}{ Country region } \\
\hline North & $11.2(6.2-19.2)$ & $1.5(0.5-4.6)$ & $28.8(22.8-35.6)$ & $58.5(51.0-65.7)$ \\
\hline Northeast & $9.7(7.7-12.2)$ & $0.5(0.2-0.9)$ & $31.0(27.6-34.7)$ & $58.8(55.0-62.5)$ \\
\hline Southeast & $9.9(8.9-10.9)$ & $0.7(0.5-1.0)$ & $28.9(27.4-30.4)$ & $60.6(58.9-62.1)$ \\
\hline South & $9.3(7.1-12.1)$ & $0.8(0.4-1.6)$ & $24.3(22.1-28.0)$ & $65.0(61.6-68.4)$ \\
\hline Central & $5.1(3.2-7.9)$ & $1.1(0.2-5.2)$ & $19.5(14.9-25.3)$ & $74.3(67.8-80.0)$ \\
\hline \multicolumn{5}{|l|}{ Working during the pandemic } \\
\hline No & $11.1(9.8-12.6)$ & $0.8(0.5-1.0)$ & $32.7(30.7-34.6)$ & $55.5(53.4-57.6)$ \\
\hline Normal routine & $9.2(7.0-11.8)$ & $0.9(0.4-2.0)$ & $21.2(18.1-24.6)$ & $68.8(65.0-72.3)$ \\
\hline Home office & $6.5(5.3-8.0)$ & $0.6(0.3-1.4)$ & $24.5(22.3-26.8)$ & $68.4(65.8-70.8)$ \\
\hline \multicolumn{5}{|c|}{ Adherence to the quarantine (months) } \\
\hline No & $8.8(6.9-11.0)$ & $0.9(0.4-1.7)$ & $22.8(20.2-25.6)$ & $67.6(64.5-70.5)$ \\
\hline$<1$ & $9.7(7.7-12.2)$ & $0.6(0.3-1.2)$ & $29.2(25.9-32.9)$ & $60.0(56.6-64.2)$ \\
\hline $1-2$ & $9.3(8.1-10.7)$ & $0.7(0.5-1.2)$ & $30.4(28.6-32.3)$ & $59.5(57.5-61.5)$ \\
\hline$>2$ & $13.1(9.8-17.2)$ & $0.9(0.4-2.1)$ & $30.3(25.0-36.1)$ & $55.8(50.0-61.5)$ \\
\hline
\end{tabular}

95\%Cl: 95\% confidence interval.

observed in 50-59 years old group (7\%) (compared to two younger age groups) and people in 1-2 months of quarantine (5\%) (compared to no quarantine group). Differences in maintenance of low and high computer/tablet use were observed for gender, age groups, academic achievement, skin color, per capita income, and working status during the pandemic, while changes reduced the proportion of "consistently low" among people who were between 1-2 months in quarantine.

Changes in prevalence of movement behaviors are presented in Table 4. Prevalence of physical inactivity ( $<150$ minutes/week), high TV-viewing time ( $\geq 4$ hours/day) and computer/tablet use $(\geq 4$ 
Table 3

Patterns of computer/tablet use according to the correlate categories during the COVID-19 pandemic. Brazil, 2020.

\begin{tabular}{|c|c|c|c|c|}
\hline & $\begin{array}{c}\text { Consistently high } \\
\%(95 \% \mathrm{Cl})\end{array}$ & $\begin{array}{l}\text { Become low } \\
\%(95 \% \mathrm{Cl})\end{array}$ & $\begin{array}{l}\text { Become high } \\
\%(95 \% \mathrm{Cl})\end{array}$ & $\begin{array}{c}\text { Consistently low } \\
\%(95 \% \mathrm{Cl})\end{array}$ \\
\hline \multicolumn{5}{|l|}{ Gender } \\
\hline Female & $34.7(33.2-36.3)$ & $4.0(3.5-4.7)$ & $22.7(21.4-24.0)$ & $38.5(36.8-40.2)$ \\
\hline Male & $47.0(44.4-49.7)$ & $3.7(2.9-4.9)$ & $19.2(17.1-21.5)$ & $30.0(27.6-32.5)$ \\
\hline \multicolumn{5}{|l|}{ Age group (years) } \\
\hline $18-29$ & $51.2(48.4-54.1)$ & $2.5(1.9-3.2)$ & $27.1(24.4-30.0)$ & $19.2(17.3-21.2)$ \\
\hline $30-39$ & $46.7(43.2-50.2)$ & $2.8(2.0-4.0)$ & $19.0(16.6-21.8)$ & $31.5(28.1-35.0)$ \\
\hline $40-49$ & $37.7(34.4-41.1)$ & $4.0(3.0-5.5)$ & $21.3(18.3-24.7)$ & $37.0(33.4-40.7)$ \\
\hline $50-59$ & $33.3(30.1-36.8)$ & $6.8(5.4-8.7)$ & $19.1(16.8-21.7)$ & $40.7(37.4-44.1)$ \\
\hline 60 & $28.7(25.2-32.5)$ & $4.4(2.8-6.7)$ & $16.3(14.1-18.9)$ & $50.6(46.8-54.5)$ \\
\hline \multicolumn{5}{|l|}{ Schooling level } \\
\hline Less than elementary school & $21.6(17.2-26.8)$ & $2.6(1.5-4.5)$ & $17.9(14.2-22.3)$ & $57.9(52.2-63.4)$ \\
\hline High school & $39.7(37.7-41.6)$ & $4.0(3.3-4.8)$ & $21.7(20.1-23.4)$ & $34.7(32.9-36.6)$ \\
\hline Higher education & $55.7(54.9-56.4)$ & $4.3(4.1-4.6)$ & $19.9(19.3-20.5)$ & $20.1(19.5-20.6)$ \\
\hline \multicolumn{5}{|l|}{ Skin color } \\
\hline White & $43.9(42.3-45.6)$ & $3.9(3.3-4.5)$ & $21.4(20.1-22.7)$ & $30.8(29.3-32.4)$ \\
\hline Black & $36.3(31.3-41.5)$ & $3.2(2.1-5.1)$ & $20.1(16.9-23.7)$ & $40.4(35.3-45.8)$ \\
\hline Brown & $38.1(35.4-40.8)$ & $4.0(3.1-5.2)$ & $20.8(18.5-21.2)$ & $37.1(34.5-39.9)$ \\
\hline Other & $46.3(38.5-54.3)$ & $4.4(2.5-7.7)$ & $22.3(15.7-30.8)$ & $26.9(19.8-35.4)$ \\
\hline \multicolumn{5}{|l|}{ Per capita income (minimun wage) } \\
\hline$<1$ & $35.3(33.1-37.6)$ & $3.4(2.7-4.3)$ & $20.7(18.9-22.7)$ & $40.6(38.2-43.0)$ \\
\hline $1-2$ & $44.4(41.2-47.7)$ & $4.0(2.9-5.5)$ & $21.2(18.7-24.0)$ & $30.3(27.4-33.3)$ \\
\hline$>2$ & $47.0(44.6-49.3)$ & $4.7(3.8-5.7)$ & $21.3(19.4-23.3)$ & $27.1(25.0-29.3)$ \\
\hline \multicolumn{5}{|l|}{ Country region } \\
\hline North & $38.2(31.5-45.4)$ & $3.4(1.9-6.0)$ & $20.1(15.3-25.8)$ & $38.3(31.2-45.9)$ \\
\hline Northeast & $39.4(35.7-43.2)$ & $4.3(3.0-6.4)$ & $20.2(17.3-23.3)$ & $36.1(32.6-39.8)$ \\
\hline Southeast & $40.5(39.0-42.1)$ & $3.9(3.3-4.6)$ & $21.8(20.5-23.2)$ & $33.8(32.2-35.4)$ \\
\hline South & $44.9(41.2-48.7)$ & $2.9(2.1-4.1)$ & $19.9(17.4-22.7)$ & $32.3(28.9-35.8)$ \\
\hline Central & $39.3(31.7-47.5)$ & $4.8(2.7-8.3)$ & $22.1(14.6-32.0)$ & $33.8(25.7-43.0)$ \\
\hline \multicolumn{5}{|l|}{ Working during the pandemic } \\
\hline No & $31.3(29.3-33.3)$ & $4.4(3.7-5.4)$ & $24.5(22.8-26.3)$ & $39.8(37.7-41.9)$ \\
\hline Normal routine & $39.1(35.6-42.7)$ & $1.6(1.1-2.3)$ & $16.0(13.4-19.1)$ & $43.3(39.7-46.9)$ \\
\hline Home office & $61.3(58.3-64.1)$ & $4.6(3.6-6.0)$ & $17.7(15.5-20.3)$ & $16.3(14.2-18.7)$ \\
\hline \multicolumn{5}{|c|}{ Adherence to the quarantine (months) } \\
\hline No & $40.1(37.0-43.2)$ & $2.6(1.9-3.5)$ & $15.7(13.6-18.0)$ & $41.7(38.6-44.8)$ \\
\hline$<1$ & $37.1(33.4-41.0)$ & $3.2(2.4-4.4)$ & $22.9(19.7-26.4)$ & $36.8(33.0-40.7)$ \\
\hline $1-2$ & $43.2(41.2-45.2)$ & $5.0(4.0-6.0)$ & $23.5(21.8-25.4)$ & $28.3(26.5-30.2)$ \\
\hline$>2$ & $36.1(30.8-41.8)$ & $3.8(2.2-6.4)$ & $18.9(15.4-23.0)$ & $41.2(35.4-47.3)$ \\
\hline
\end{tabular}

95\%Cl: 95\% confidence interval.

hours/day) increased in all population sub-groups. Increases in physical inactivity varied from $13 \%$ (lower schooling level) to 37\% (higher income). Increases in high TV viewing varied from 121\% (> 60 years) to $613 \%$ (18-29 years), and the increases of high computer/tablet use ranged from $20 \%$ (people at home office) to $56 \%$ (no working during the pandemic) 
Table 4

Changes in prevalence of physical inactivity and sedentary behavior according to correlates, before and during the COVID-19 pandemic. Brazil, 2020.

\begin{tabular}{|c|c|c|c|c|c|c|c|c|c|}
\hline & \multicolumn{3}{|c|}{$\begin{array}{c}<150 \text { minutes/week of physical } \\
\text { activity }\end{array}$} & \multicolumn{3}{|c|}{$\geq 4$ hours/day of TV viewing } & \multicolumn{3}{|c|}{$\begin{array}{l}\geq 4 \text { hours/day of computer/ } \\
\text { tablet }\end{array}$} \\
\hline & $\begin{array}{c}\text { Before } \\
\%(95 \% \mathrm{Cl})\end{array}$ & $\begin{array}{c}\text { During } \\
\%(95 \% \mathrm{Cl})\end{array}$ & $\% \Delta$ & $\begin{array}{c}\text { Before } \\
\%(95 \% \mathrm{Cl})\end{array}$ & $\begin{array}{c}\text { During } \\
\%(95 \% \mathrm{Cl})\end{array}$ & $\% \Delta$ & $\begin{array}{c}\text { Before } \\
\%(95 \% \mathrm{Cl})\end{array}$ & $\begin{array}{c}\text { During } \\
\%(95 \% \mathrm{Cl})\end{array}$ & $\% \Delta$ \\
\hline \multicolumn{10}{|l|}{ Gender } \\
\hline Female & $\begin{array}{c}72.2 \\
(70.7-73.7)\end{array}$ & $\begin{array}{c}89.7 \\
(88.7-90.6)\end{array}$ & 24 & $\begin{array}{c}10.2 \\
(9.1-11.4)\end{array}$ & $\begin{array}{c}39.4 \\
(37.7-41.1)\end{array}$ & 286 & $\begin{array}{c}38.8 \\
(37.2-40.4)\end{array}$ & $\begin{array}{c}57.4 \\
(55.7-59.1)\end{array}$ & 48 \\
\hline Male & $\begin{array}{c}67.3 \\
(64.8-69.6)\end{array}$ & $\begin{array}{c}85.6 \\
(83.7-87.3)\end{array}$ & 27 & $\begin{array}{c}10.3 \\
(8.8-12.1)\end{array}$ & $\begin{array}{c}35.8 \\
(33.4-38.4)\end{array}$ & 248 & $\begin{array}{c}50.8 \\
(48.1-53.4)\end{array}$ & $\begin{array}{c}66.2 \\
(63.7-68.7)\end{array}$ & 30 \\
\hline \multicolumn{10}{|l|}{ Age group (years) } \\
\hline $18-29$ & $\begin{array}{c}68.0 \\
(65.3-70.5)\end{array}$ & $\begin{array}{c}88.7 \\
(87.0-90.2)\end{array}$ & 30 & $\begin{array}{c}4.2 \\
(3.2-5.5)\end{array}$ & $\begin{array}{c}30.7 \\
(28.1-33.3)\end{array}$ & 631 & $\begin{array}{c}53.7 \\
(50.8-56.6)\end{array}$ & $\begin{array}{c}78.4 \\
(76.2-80.3)\end{array}$ & 46 \\
\hline $30-39$ & $\begin{array}{c}69.3 \\
(66.0-72.4)\end{array}$ & $\begin{array}{c}88.9 \\
(86.6-90.9)\end{array}$ & 31 & $\begin{array}{c}7.3 \\
(5.9-9.1)\end{array}$ & $\begin{array}{c}37.9 \\
(34.6-41.4)\end{array}$ & 419 & $\begin{array}{c}49.5 \\
(46.0-53.0)\end{array}$ & $\begin{array}{c}65.7 \\
(62.1-69.1)\end{array}$ & 33 \\
\hline $40-49$ & $\begin{array}{c}72.3 \\
(69.1-75.4)\end{array}$ & $\begin{array}{c}88.1 \\
(85.4-90.3)\end{array}$ & 22 & $\begin{array}{c}10.2 \\
(7.9-13.1)\end{array}$ & $\begin{array}{c}39.3 \\
(35.8-43.0)\end{array}$ & 285 & $\begin{array}{c}41.7 \\
(38.3-45.2)\end{array}$ & $\begin{array}{c}59.0 \\
(55.2-62.6)\end{array}$ & 42 \\
\hline $50-59$ & $\begin{array}{c}70.3 \\
(67.3-73.2)\end{array}$ & $\begin{array}{c}86.0 \\
(83.6-88.1)\end{array}$ & 22 & $\begin{array}{c}13.9 \\
(11.8-16.3)\end{array}$ & $\begin{array}{c}42.0 \\
(38.7-45.4)\end{array}$ & 202 & $\begin{array}{c}40.2 \\
(36.8-43.6)\end{array}$ & $\begin{array}{c}52.4 \\
(49.0-55.8)\end{array}$ & 30 \\
\hline 60 & $\begin{array}{c}70.2 \\
(66.5-73.7)\end{array}$ & $\begin{array}{c}86.1 \\
(83.1-88.7)\end{array}$ & 23 & $\begin{array}{c}18.8 \\
(15.8-22.2)\end{array}$ & $\begin{array}{c}41.6 \\
(37.8-45.4)\end{array}$ & 121 & $\begin{array}{c}33.1 \\
(29.4-37.0)\end{array}$ & $\begin{array}{c}45.0 \\
(41.2-48.9)\end{array}$ & 36 \\
\hline \multicolumn{10}{|l|}{ Schooling level } \\
\hline Less than elementary school & $\begin{array}{c}78.8 \\
(73.8-83.1)\end{array}$ & $\begin{array}{c}89.1 \\
(85.1-92.2)\end{array}$ & 13 & $\begin{array}{c}15.6 \\
(11.7-20.5)\end{array}$ & $\begin{array}{c}41.2 \\
(35.8-46.9)\end{array}$ & 164 & $\begin{array}{c}24.2 \\
(19.6-29.4)\end{array}$ & $\begin{array}{c}39.5 \\
(34.1-45.1)\end{array}$ & 63 \\
\hline High school & $\begin{array}{c}70.8 \\
(69.0-72.5)\end{array}$ & $\begin{array}{c}88.2 \\
(86.9-89.4)\end{array}$ & 25 & $\begin{array}{c}10.6 \\
(9.4-11.8)\end{array}$ & $\begin{array}{c}38.5 \\
(36.6-40.4)\end{array}$ & 263 & $\begin{array}{c}43.6 \\
(41.7-45.6)\end{array}$ & $\begin{array}{c}61.3 \\
(59.4-63.2)\end{array}$ & 41 \\
\hline Higher education & $\begin{array}{c}60.8 \\
(60.0-61.5)\end{array}$ & $\begin{array}{c}85.1 \\
(84.5-85.6)\end{array}$ & 40 & $\begin{array}{c}6.0 \\
(5.7-6.4)\end{array}$ & $\begin{array}{c}32.2 \\
(31.5-32.9)\end{array}$ & 437 & $\begin{array}{c}60.0 \\
(59.3-60.7)\end{array}$ & $\begin{array}{c}75.6 \\
(75.0-76.2)\end{array}$ & 26 \\
\hline \multicolumn{10}{|l|}{ Skin color } \\
\hline White & $\begin{array}{c}68.1 \\
(66.6-69.6)\end{array}$ & $\begin{array}{c}87.3 \\
(86.2-88.4)\end{array}$ & 28 & $\begin{array}{c}9.5 \\
(8.6-10.5)\end{array}$ & $\begin{array}{c}35.4 \\
(33.9-37.0)\end{array}$ & 273 & $\begin{array}{c}47.8 \\
(46.1-49.4)\end{array}$ & $\begin{array}{c}65.3 \\
(63.7-66.9)\end{array}$ & 37 \\
\hline Black & $\begin{array}{c}69.6 \\
(64.9-73.9)\end{array}$ & $\begin{array}{c}85.6 \\
(81.6-88.9)\end{array}$ & 23 & $\begin{array}{c}13.0 \\
(9.8-17.2)\end{array}$ & $\begin{array}{c}42.5 \\
(37.4-47.8)\end{array}$ & 227 & $\begin{array}{c}39.5 \\
(34.5-44.7)\end{array}$ & $\begin{array}{c}56.3 \\
(51.0-61.5)\end{array}$ & 43 \\
\hline Brown & $\begin{array}{c}71.6 \\
(69.0-74.1)\end{array}$ & $\begin{array}{c}88.5 \\
(86.6-90.1)\end{array}$ & 24 & $\begin{array}{c}10.6 \\
(9.0-12.5)\end{array}$ & $\begin{array}{c}39.1 \\
(36.3-41.9)\end{array}$ & 269 & $\begin{array}{c}42.1 \\
(39.4-44.9)\end{array}$ & $\begin{array}{c}58.8 \\
(56.0-61.6)\end{array}$ & 40 \\
\hline Other & $\begin{array}{c}70.2 \\
(63.3-76.3)\end{array}$ & $\begin{array}{c}88.4 \\
(84.3-91.6)\end{array}$ & 26 & $\begin{array}{c}7.9 \\
(4.2-14.5)\end{array}$ & $\begin{array}{c}36.5 \\
(28.7-45.1)\end{array}$ & 362 & $\begin{array}{c}50.7 \\
(42.6-58.9)\end{array}$ & $\begin{array}{c}68.7 \\
(60.3-76.0)\end{array}$ & 36 \\
\hline \multicolumn{10}{|c|}{ Per capita income (minimum wages) } \\
\hline$<1$ & $\begin{array}{c}73.3 \\
(71.0-75.4)\end{array}$ & $\begin{array}{c}89.1 \\
(87.5-90.6)\end{array}$ & 22 & $\begin{array}{c}10.4 \\
(9.1-11.9)\end{array}$ & $\begin{array}{c}37.8 \\
(35.6-40.1)\end{array}$ & 263 & $\begin{array}{c}38.7 \\
(36.4-41.0)\end{array}$ & $\begin{array}{c}56.0 \\
(53.6-58.4)\end{array}$ & 45 \\
\hline $1-2$ & $\begin{array}{c}72.7 \\
(69.9-75.3)\end{array}$ & $\begin{array}{c}89.2 \\
(87.1-90.9)\end{array}$ & 23 & $\begin{array}{c}11.1 \\
(8.9-13.7)\end{array}$ & $\begin{array}{c}39.4 \\
(36.2-42.6)\end{array}$ & 255 & $\begin{array}{c}48.4 \\
(45.2-51.7)\end{array}$ & $\begin{array}{c}65.6 \\
(62.5-68.7)\end{array}$ & 36 \\
\hline$>2$ & $\begin{array}{c}61.1 \\
(58.8-63.4)\end{array}$ & $\begin{array}{c}83.9 \\
(82.1-85.4)\end{array}$ & 37 & $\begin{array}{c}9.3 \\
(8.0-10.7)\end{array}$ & $\begin{array}{c}36.0 \\
(33.7-38.3)\end{array}$ & 287 & $\begin{array}{c}51.6 \\
(49.2-54.0)\end{array}$ & $\begin{array}{c}68.2 \\
(65.9-70.5)\end{array}$ & 32 \\
\hline
\end{tabular}

(continues) 


\begin{tabular}{|c|c|c|c|c|c|c|c|c|c|}
\hline & \multicolumn{3}{|c|}{$\begin{array}{c}<150 \text { minutes/week of physical } \\
\text { activity }\end{array}$} & \multicolumn{3}{|c|}{$\geq 4$ hours/day of TV viewing } & \multicolumn{3}{|c|}{$\begin{array}{c}\geq 4 \text { hours/day of computer/ } \\
\text { tablet }\end{array}$} \\
\hline & $\begin{array}{c}\text { Before } \\
\%(95 \% \mathrm{Cl})\end{array}$ & $\begin{array}{c}\text { During } \\
\%(95 \% \mathrm{Cl})\end{array}$ & $\% \Delta$ & $\begin{array}{c}\text { Before } \\
\%(95 \% \mathrm{Cl})\end{array}$ & $\begin{array}{c}\text { During } \\
\%(95 \% \mathrm{Cl})\end{array}$ & $\% \Delta$ & $\begin{array}{c}\text { Before } \\
\%(95 \% \mathrm{Cl})\end{array}$ & $\begin{array}{c}\text { During } \\
\%(95 \% \mathrm{Cl})\end{array}$ & $\% \Delta$ \\
\hline \multicolumn{10}{|l|}{ Country region } \\
\hline North & $\begin{array}{c}71.5 \\
(64.9-77.3)\end{array}$ & $\begin{array}{c}89.1 \\
(83.7-92.8)\end{array}$ & 25 & $\begin{array}{c}12.7 \\
(7.5-20.6)\end{array}$ & $\begin{array}{c}40.0 \\
(32.9-47.5)\end{array}$ & 215 & $\begin{array}{c}41.6 \\
(34.8-48.8)\end{array}$ & $\begin{array}{c}58.3 \\
(50.8-65.4)\end{array}$ & 40 \\
\hline Northeast & $\begin{array}{c}67.2 \\
(63.4-70.8)\end{array}$ & $\begin{array}{c}87.9 \\
(85.1-90.2)\end{array}$ & 31 & $\begin{array}{c}10.2 \\
(8.2-12.7)\end{array}$ & $\begin{array}{c}40.8 \\
(37.0-44.6)\end{array}$ & 300 & $\begin{array}{c}43.7 \\
(40.0-47.6)\end{array}$ & $\begin{array}{c}59.5 \\
(55.8-63.2)\end{array}$ & 36 \\
\hline Southeast & $\begin{array}{c}69.2 \\
(67.7-70.6)\end{array}$ & $\begin{array}{c}86.3 \\
(85.1-87.4)\end{array}$ & 25 & $\begin{array}{c}10.6 \\
(9.6-11.7)\end{array}$ & $\begin{array}{c}38.7 \\
(37.1-40.3)\end{array}$ & 265 & $\begin{array}{c}44.4 \\
(42.8-46.0)\end{array}$ & $\begin{array}{c}62.3 \\
(60.7-63.9)\end{array}$ & 40 \\
\hline South & $\begin{array}{c}71.9 \\
(68.4-75.0)\end{array}$ & $\begin{array}{c}89.2 \\
(86.6-91.3)\end{array}$ & 24 & $\begin{array}{c}10.1 \\
(7.8-12.9)\end{array}$ & $\begin{array}{c}34.2 \\
(30.8-37.8)\end{array}$ & 237 & $\begin{array}{c}47.8 \\
(44.1-51.6)\end{array}$ & $\begin{array}{c}64.8 \\
(61.2-68.2)\end{array}$ & 36 \\
\hline Central & $\begin{array}{c}77.8 \\
(71.9-82.8)\end{array}$ & $\begin{array}{c}92.0 \\
(88.3-94.6)\end{array}$ & 18 & $\begin{array}{c}6.2 \\
(3.9-9.7)\end{array}$ & $\begin{array}{c}24.6 \\
(19.2-30.9)\end{array}$ & 297 & $\begin{array}{c}44.1 \\
(36.0-52.5)\end{array}$ & $\begin{array}{c}61.4 \\
(52.4-69.7)\end{array}$ & 39 \\
\hline \multicolumn{10}{|c|}{ Working during the pandemic } \\
\hline No & $\begin{array}{c}69.9 \\
(67.8-71.8)\end{array}$ & $\begin{array}{c}87.8 \\
(86.4-89.1)\end{array}$ & 26 & $\begin{array}{c}11.9 \\
(10.5-13.3)\end{array}$ & $\begin{array}{c}43.8 \\
(41.7-45.9)\end{array}$ & 268 & $\begin{array}{c}35.7 \\
(33.7-37.8)\end{array}$ & $\begin{array}{c}55.8 \\
(53.6-57.9)\end{array}$ & 56 \\
\hline Normal routine & $\begin{array}{c}70.6 \\
(67.3-73.6)\end{array}$ & $\begin{array}{c}88.9 \\
(86.5-90.9)\end{array}$ & 26 & $\begin{array}{c}10.1 \\
(7.9-12.8)\end{array}$ & $\begin{array}{c}30.3 \\
(26.8-34.1)\end{array}$ & 200 & $\begin{array}{c}40.7 \\
(37.2-44.3)\end{array}$ & $\begin{array}{c}55.2 \\
(51.5-58.7)\end{array}$ & 36 \\
\hline Home office & $\begin{array}{c}69.2 \\
(66.7-71.6)\end{array}$ & $\begin{array}{c}86.6 \\
(84.6-88.4)\end{array}$ & 25 & $\begin{array}{c}7.2 \\
(5.9-8.7)\end{array}$ & $\begin{array}{c}31.0 \\
(28.6-33.5)\end{array}$ & 331 & $\begin{array}{c}65.9 \\
(63.0-68.7)\end{array}$ & $\begin{array}{c}79.0 \\
(76.5-81.4)\end{array}$ & 20 \\
\hline \multicolumn{10}{|c|}{$\begin{array}{l}\text { Adherence to the quarantine } \\
\text { (months) }\end{array}$} \\
\hline No & $\begin{array}{c}69.7 \\
(66.7-72.6)\end{array}$ & $\begin{array}{c}87.4 \\
(85.0-89.4)\end{array}$ & 25 & $\begin{array}{c}9.6 \\
(7.7-11.9)\end{array}$ & $\begin{array}{c}31.5 \\
(28.6-34.6)\end{array}$ & 228 & $\begin{array}{c}42.7 \\
(39.6-45.8)\end{array}$ & $\begin{array}{c}55.8 \\
(52.6-58.8)\end{array}$ & 31 \\
\hline$<1$ & $\begin{array}{c}72.4 \\
(68.8-75.7)\end{array}$ & $\begin{array}{c}90.3 \\
(88.2-92.1)\end{array}$ & 25 & $\begin{array}{c}10.3 \\
(8.3-12.8)\end{array}$ & $\begin{array}{c}38.9 \\
(35.2-42.8)\end{array}$ & 278 & $\begin{array}{c}40.3 \\
(36.6-44.2)\end{array}$ & $\begin{array}{c}60.0 \\
(56.0-63.8)\end{array}$ & 49 \\
\hline $1-2$ & $\begin{array}{c}69.0 \\
(67.2-70.8)\end{array}$ & $\begin{array}{c}86.9 \\
(85.4-88.2)\end{array}$ & 26 & $\begin{array}{c}10.0 \\
(8.8-11.4)\end{array}$ & $\begin{array}{c}39.7 \\
(37.7-41.7)\end{array}$ & 297 & $\begin{array}{c}48.2 \\
(46.2-50.2)\end{array}$ & $\begin{array}{c}66.8 \\
(64.8-68.7)\end{array}$ & 39 \\
\hline$>2$ & $\begin{array}{c}68.3 \\
(62.4-73.8)\end{array}$ & $\begin{array}{c}87.3 \\
(83.0-90.6)\end{array}$ & 28 & $\begin{array}{c}13.4 \\
(10.6-18.1)\end{array}$ & $\begin{array}{c}43.3 \\
(37.7-49.2)\end{array}$ & 223 & $\begin{array}{c}39.9 \\
(34.4-45.6)\end{array}$ & $\begin{array}{c}55.0 \\
(49.0-60.8)\end{array}$ & 38 \\
\hline
\end{tabular}

95\%Cl: 95\% confidence interval.

\section{Discussion}

\section{Main finding of this study}

Our main results showed that the prevalence of $\geq 4$ hours/day of TV-viewing increased 266\% during the pandemic among Brazilian adults, while physical inactivity and $\geq 4$ hours/day of computer/ tablet use increased $26 \%$ and $38 \%$, respectively. We observed that the prevalence of these behaviors increased in all population groups. However, groups presented different proportions of changed behaviors ("become low active/high" and "become active/low"), and differences were observed in between-groups comparisons before and during the COVID-19 pandemic. Thus, we observed an effect of quarantine on the prevalence of high TV-viewing time (affected by working status and adherence to stricter quarantine measures), but not on computer/tablet use and physical inactivity, which increased similarly between groups. 


\section{What is already known on this topic}

Other studies were conducted to identify the effect of the COVID-19 pandemic on movement behaviors worldwide $10,16,17,18,19,20,21$. However, to the best of our knowledge, this is the first survey with a nationwide sampling, which strengthen the prevalence analyses.

\section{What this study adds}

Although these results confirm previous hypotheses of increases in physical inactivity and sedentary behaviours with quarantine measures, we observed that the prevalence of high TV viewing increases more than three time during the pandemic and the increase was substantially higher among youngers (18-29 years: $631 \%$ and $30-39$ years: $419 \%$ ) compared to the older adults (> 60 years: $121 \%$ ), and among the higher academic achievement (437\%) compared to the lower academic achievement group (164\%). In these cases, even with reductions on the differences between-groups, the prevalence of high TV-viewing remains higher among older adults and lower academic achievement groups during the pandemic.

In other way, there were no differences in the prevalence of high TV-viewing time between country regions before the pandemic, but changes due to the COVID-19 pandemic increased the prevalence in the North, the Northeast, and the Southeast regions when comparing with the Central during the pandemic. These findings suggest that Brazilian adults become much more sedentary during the COVID-19 pandemic. TV-viewing is a classical proxy of sedentary behavior, being one of the types of behavior more associated with harmful effects on cardiovascular and mental health 15,22.

Increases in the prevalence of high computer and tablet use seemed more widespread, and these changes during the pandemic were similar among population groups. Younger participants presented higher relative increase than older adults. On the other hand, people who were at home office during the pandemic already had higher prevalence of computer/tablet use before it, and even with lower relative increases, their prevalence remained higher than among people who were not working or remained their normal working routines during the pandemic. In quarantine times, computer and tablet are options to keep work-related and study activities as well as social interaction, highlighting computer/tablet use as a significant manifestation of sedentary behaviors during this period. Therefore, recommendations about the screens use, such as breaking up long bouts of sedentary behaviors 23 or avoid their use until late at night 24 , should be disseminated.

The prevalence of physical inactivity was already higher in comparison with TV viewing time and computer use before the COVID-19 pandemic, however it was similar to the last Brazilian National Health Survey 2. Generally, higher increases in physical inactivity prevalence were observed among more active groups before the pandemic. For example, people with higher schooling level presented a higher increase in the prevalence of physical inactivity and differences between lower and higher school levels groups disappeared during the COVID-19 pandemic. Similar results were found on per capita income, in which the difference between higher and lower per capita income reduced during the COVID-19 pandemic. Moreover, we observed no effect in working status during the pandemic and the adherence to the quarantine on physical inactivity prevalence. These findings are worrying, considering the benefits of physical activity for physical and mental health. While the Global Physical Activity Action Plan 2018-2030 3 targets reductions of $15 \%$ by 2025 and 30\% by 2030, we observed an increase of $26 \%$ after around two months of quarantine in Brazil, which suggests further studies and actions are necessary considering the unpredictable scenario ahead. The World Health Organization and scientific societies launched some initiatives, such as educational materials on the relevance of exercises at home ${ }^{8}$. Studies have suggested the use of text messages and phone calls as an effective and viable contact channel between healthcare professionals and patients 8 . It is necessary to mobilize governments and civil society to mitigate these results. 


\section{Limitations of this study}

Limitations of the current study include the online survey. Although our sampling process was planned to include people of different social strata and the sample was weighted according characteristics from the PNAD to let the sample nationally representative, this type of survey limits the participation of people with lower socioeconomic position without access to Internet. Also, the retrospective design can produce recall bias. However, this is the first nationwide survey examining the changes in the prevalence of movement behaviours during the COVID-19 pandemic among Brazilian adults, which could foment policies to promote active habits during the present and next quarantine times.

\section{Conclusion}

The prevalence of physical inactivity and sedentary behaviors increased during COVID-19 pandemic among Brazilian adults. Although all population groups were affected, higher increases in the prevalence of high TV-viewing time were found among younger adults and those who adhered to more stricter measures of quarantine. During the pandemic, around $88 \%$ of adults are not meeting international guidelines for physical activity, 38\% are spending $\geq 4$ hours/day watching TV and $62 \%$ are $\geq 4$ hours/day in computer or tablet use. Considering that the COVID-19 pandemic is not in its peak in Brazil in the period of this survey, population should be continuously surveyed, and interventions must be developed to promote physical activity and to reduce sedentary behaviors during the quarantine period. 


\section{Contributors}

All authors contributed to the study conception and design. Material preparation and data analysis were performed by D. R. Silva, A. O. Werneck and C. L. Szwarcwald. The first draft of the manuscript was written by D. R. P. Silva and all authors commented on previous versions of the manuscript.

\section{Additional informations}

ORCID: Danilo Rodrigues Pereira da Silva (00000003-3995-4795); André Oliveira Werneck (00000002-9166-4376); Deborah Carvalho Malta (00000002-8214-5734); Paulo Roberto Borges de Souza Júnior (0000-0002-8142-4790); Luiz Otávio Azevedo (0000-0002-4876-5948); Marilisa Berti de Azevedo Barros (0000-0003-3974-195X); Celia Landmann Szwarcwald (0000-0002-7798-2095).

\section{Acknowledgments}

A. O. Werneck is supported by the São Paulo State Research Foundation (FAPESP) with a PhD scholarship (FAPESP process: 2019/24124-7). D. C. Malta (process: 308250/2017-6), M. B. A. Barros (process: 303241/2019-5) and C. L. Szwarcwald (process: 310318/2020-3) are supported by the Brazilian National Research Council (CNPq), which funded the productivity scholarship. This paper presents an independent research. The views expressed in this publication are those of the authors and they do not necessary those of the acknowledged institutions.

\section{References}

1. Hallal PC, Andersen LB, Bull FC, Guthold R, Haskell W, Ekelund U. Global physical activity levels: surveillance progress, pitfalls, and prospects. Lancet 2012; 380:247-57.

2. Werneck AO, Baldew SS, Miranda JJ, Díaz Arnesto O, Stubbs B, Silva DR. Physical activity and sedentary behavior patterns and sociodemographic correlates in 116,982 adults from six South American countries: the South American physical activity and sedentary behavior network (SAPASEN). Int J Behav Nutr Phys Act 2019; 16:68.

3. World Health O. Global action plan on physical activity 2018-2030: more active people for a healthier world. Geneva: World Health Organization; 2018.

4. Cucinotta D, Vanelli M. WHO declares COVID-19 a pandemic. Acta Biomed 2020; 91:157-60.

5. World Health Organization. Coronavirus disease 2019 (COVID-19): situation report - 73. Geneva: World Health Organization; 2020.

6. Chen P, Mao L, Nassis GP, Harmer P, Ainsworth BE, Li F. Coronavirus disease (COVID-19): the need to maintain regular physical activity while taking precautions. J Sport Health Sci 2020; 9:103-4.

7. Sallis JF, Adlakha D, Oyeyemi A, Salvo D. An international physical activity and public health research agenda to inform COVID-19 policies and practices. J Sport Health Sci 2020; 9:328-34.

8. Peçanha T, Goessler KF, Roschel H, Gualano B. Social isolation during the COVID-19 pandemic can increase physical inactivity and the global burden of cardiovascular disease. Am J Physiol Heart Circ Physiol 2020; 318:H14416.

9. Narici M, De Vito G, Franchi M, Paoli A, Moro $\mathrm{T}$, Marcolin $\mathrm{G}$, et al. Impact of sedentarism due to the COVID-19 home confinement on neuromuscular, cardiovascular and metabolic health: physiological and pathophysiological implications and recommendations for physical and nutritional countermeasures. Eur J Sport Sci 2020. [Online ahead of print].

10. Schuch F, Bulzing R, Meyer J, Vancampfort D, Firth J, Stubbs B, et al. Associations of moderate to vigorous physical activity and sedentary behavior with depressive and anxiety symptoms in self-isolating people during the COVID-19 pandemic: a cross-sectional survey in Brazil. Psychiatry Res 2020; 292:113339.

11. Duggal NA, Niemiro G, Harridge SDR, Simpson RJ, Lord JM. Can physical activity ameliorate immunosenescence and thereby reduce age-related multi-morbidity? Nat Rev Immunol 2019; 19:563-72.

12. Simpson RJ, Katsanis E. The immunological case for staying active during the COVID-19 pandemic. Brain Behav Immun 2020; 87:6-7. 
13. Laddu DR, Lavie CJ, Phillips SA, Arena R. Physical activity for immunity protection: inoculating populations with healthy living medicine in preparation for the next pandemic. Prog Cardiovasc Dis 2020; [Epub ahead of print].

14. Szwarcwald CL, Souza Júnior PRB, Damacena GN, Malta DC, Barros MBA, Romero DE, et al. ConVid - Pesquisa de Comportamentos pela Internet durante a pandemia de COVID-19 no Brasil: concepção e metodologia de aplicação. Cad Saúde Pública 2021; 37:e00268320.

15. Patterson R, McNamara E, Tainio M, de Sá $\mathrm{TH}$, Smith AD, Sharp SJ, et al. Sedentary behaviour and risk of all-cause, cardiovascular and cancer mortality, and incident type 2 diabetes: a systematic review and dose response meta-analysis. Eur J Epidemiol 2018; 33:81129.

16. Ammar A, Brach M, Trabelsi K, Chtourou $\mathrm{H}$, Boukhris O, Masmoudi L, et al. Effects of COVID-19 home confinement on eating behaviour and physical activity: results of the ECLB-COVID19 International Online Survey. Nutrients 2020; 12:1583.

17. Gallè F, Sabella EA, Da Molin G, De Giglio O, Caggiano G, Di Onofrio V, et al. Understanding knowledge and behaviors related to CoViD-19 epidemic in Italian undergraduate students: the EPICO Study. Int J Environ Res Public Health 2020; 17:3481.

18. Goethals L, Barth N, Guyot J, Hupin D, Celarier T, Bongue B. Impact of home quarantine on physical activity among older adults living at home during the COVID-19 pandemic: qualitative interview study. JMIR Aging 2020; 3:e19007.
19. Lesser IA, Nienhuis CP. The impact of COVID-19 on physical activity behavior and well-being of Canadians. Int J Environ Res Public Health 2020; 17:3899.

20. Dunton G, Wang S, Do B, Courtney J. Early effects of the COVID-19 pandemic on physical activity in US adults. Cambridge Open Engage 2020; 15 may. https:// www.cambridge.org/engage/coe/articledetails/5ebc9163597aba001941b1fe.

21. Meyer J, McDowell C, Lansing J, Brower C, Smith L, Tully M, et al. Changes in physical activity and sedentary behaviour due to the COVID-19 outbreak and associations with mental health in 3,052 US adults. Cambridge Open Engage 2020; 12 may. https:// www.cambridge.org/engage/coe/articledetails/5eb2056d7a31fc00183d05db.

22. Werneck AO, Oyeyemi AL, Szwarcwald CL, Vancampfort D, Silva DR. Associations between TV viewing and depressive symptoms among 60,202 Brazilian adults: The Brazilian national health survey. J Affect Disord 2018; 236:23-30

23. Piercy KL, Troiano RP, Ballard RM, Carlson SA, Fulton JE, Galuska DA, et al. The Physical Activity Guidelines for Americans. JAMA 2018; 320:2020-8.

24. Šmotek M, Fárková E, Manková D, Kopřivová J. Evening and night exposure to screens of media devices and its association with subjectively perceived sleep: should "light hygiene" be given more attention? Sleep Health 2020; 6:498-505 


\section{Resumo}

O estudo buscou analisar mudanças na prevalência de inatividade física e comportamento sedentário de acordo com correlatos durante a pandemia da COVID-19 entre adultos brasileiros. Foi realizado um inquérito retrospectivo online, de base nacional, com 39.693 adultos brasileiros. Foram relatadas antes e durante a pandemia: atividade física (frequência semanal e duração diária; ponto de corte de 150 minutos/semana), tempo em TV e computador/tablet (duração diária; ponto de corte de 4 horas/dia). Os correlatos foram sexo, faixa etária, escolaridade, cor da pele, renda per capita, macrorregião, situação de trabalho durante o isolamento social e adesão ao isolamento. Foram utilizadas estatísticas descritivas. A prevalência de inatividade física, tempo de TV e tempo de computador/tablet aumentaram 26\%, 266\% e $38 \%$, respectivamente, durante a pandemia. Os aumentos na inatividade física e no uso de computador/tablet foram mais prevalentes em geral, mas o aumento na prevalência de tempo elevado na frente da televisão foi maior entre adultos mais jovens (660\%), com maior escolaridade (437\%) $e$ aqueles que trabalhavam em home office (331\%). A prevalência de inatividade física e de comportamento sedentário aumentou em todos os subgrupos da população brasileira durante a pandemia da COVID-19.

Comportamentos Relacionados com a Saúde; Exercício Físico; Estilo de Vida Saudável; COVID-19

\section{Resumen}

El objetivo de este estudio fue analizar los cambios en la prevalencia de inactividad física y comportamientos sedentarios, según correlaciones durante la pandemia de COVID-19, entre adultos brasilenos. Se realizó una encuesta nacional retrospectiva con 39.693 adultos brasileños. Se informó de actividad física (frecuencia semanal y duración diaria; punto de corte de 150 minutos/semana), ver TV y el uso de computadora/tablet (duración diaria; punto de corte de 4 horas/dia) antes y durante el periodo pandémico. Las correlaciones fueron: sexo, grupo de edad, logros académicos más altos, color de piel, ingresos per cápita, macrorregión del país, estatus laboral durante la cuarentena, y adherencia a la cuarentena. Se usaron estadísticas descriptivas. La prevalencia de inactividad física, ver la TV durante mucho tiempo y el uso de computadora/tablet, incrementó, respectivamente, un 26\%, 266\% y 38\% durante la pandemia. Al igual que el incremento en inactividad física, y que el uso de la computadora/tablet era el más extendido, se observaron incrementos más altos en la prevalencia de ver más la TV entre los jóvenes adultos (660\%), con un nivel de educación más alto (437\%) $y$ entre quienes estaban teletrabajando (331\%). La prevalencia de inactividad física y comportamientos sedentarios se incrementaron en todos los subgrupos de población durante la pandemia de COVID-19 en Brasil.

Conductas Relacionadas con la Salud; Exercício Físico; Estilo de Vida Saludable; COVID-19
Submitted on $27 / \mathrm{Jul} / 2020$

Final version resubmitted on 01/Nov/2020

Approved on 11/Dec/2020 